\title{
7. Zusammenfassung und zukünftige Perspektiven
}

Zielsetzung der Arbeit war die Entwicklung eines Handlungskonzepts für traumatisierte, unbegleitete, minderjährige Flüchtlinge in Jugendhilfeeinrichtungen, um diesen Personen damit bestmögliche Chancen für ihre personelle und existentielle Zukunft zu geben.

Nach der Untersuchung der grundlegenden Faktoren im Zusammenhang mit der Migrationssituation, der Flucht, der Traumatisierung, den entwicklungspsychologischen Erfordernissen sowie den möglichen Ressourcen seitens der Resilienzforschung und unter dem Aspekt des Phänomens der Übergangswelten wurden diese kritisch hinterfragt und hinsichtlich der beabsichtigten Aufgabenstellung überprüft.

Unklare und sogar widersprüchliche rechtliche Vorgaben wurden aufgezeigt und notwendige Aktualisierungen gesetzlicher Anordnungen eingefordert.

Die Rahmenbedingungen der Einrichtungen wurden hinsichtlich ihrer Voraussetzungen für die besonderen Erfordernisse des Personenkreises hinterfragt.

Das Konzept wurde in seinem zeitlichen und strukturellen Ablauf vorgestellt und dabei die jeweils vordringlichen Bedürfnisse der minderjährigen Flüchtlinge deutlich gemacht, die einzelnen Ziele formuliert und die jeweiligen Anforderungen an die sozialpädagogischen Fachkräfte herausgestellt.

$\mathrm{Da}$ in der Regel in den betreffenden Einrichtungen bisher eine Aufnahme in bestehende Gruppen ohne spezielle, handlungsleitende Vorgaben erfolgt, besteht ein dringender Handlungsbedarf zur Konkretisierung dieses Vorhabens.

Es ist zu wünschen, dass das nun erarbeitete Konzept in den Jugendhilfeeinrichtungen als Grundlage für dieses neue, sozialpädagogische Aufgabenfeld aufgenommen und die Mitarbeiter dafür sensibilisiert werden.

Durch die übersichtliche Gestaltung des zeitlichen und organisatorischen Ablaufs wird ein konstruktiver Beitrag zur Umsetzung und damit zu einer Intensivierung dieses Arbeitsfeldes geleistet.

In den jeweiligen Teams mit den beteiligten Mitarbeitern wird der künftige Auftrag sein, die Praxistauglichkeit des Konzepts gemeinsam zu erproben und es weiter zu entwickeln.

Damit erhält der Leitspruch vieler Jugendhilfeeinrichtungen: „Keiner darf verloren gehen" eine neue, der aktuellen Herausforderung gemäße Interpretation, indem in diesem aktuellen, sozialpolitischen Brennpunkt neue Perspektiven erschlossen werden.

Vor allen Dingen aber geht es darum, den Appell des Aufnahmelandes Deutschland und Europas zur humanitären Hilfe für den Personenkreis traumatisierter minderjähriger Flüchtlinge mit zielgerichteten Methoden und einem maßgeschneiderten Konzept der Sozialen Arbeit konstruktiv in die Realität umzusetzen. 